\title{
The Principle of Priority in a Hierarchical System With Inner Dependence
}

\author{
Shubo $\cdot \mathrm{Xu}$ \\ The College of West Virginia \\ Beckley, WV 25801, USA
}

\author{
Hui Wang \\ Tianjin Industry Development Company \\ Tianjin, China
}

After we classified feedback systems in the AHP according to supermatrices, deriving the Limiting Impact Priority (LIP) for the hierarchical systems with inner dependence has been discussed in this paper with a numerical example.

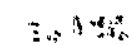

1. Introduction

When people apply the AHP in decision making, they always break the decision problem into individual criteria, subcriteria, factors, players, alternatives, and etc., group them to form different levels, and construct a hierarchical structure to demonstrate the relationship among the elements and group. Constructing hierarchical stnucture is the most important step in the AHP. Then people may use the 1-9 ratio scale to make pairwise comparison to derive a local priority of elements in the same level with respect to a higher level element, or simply give the local priority by employ their physical measurements. The principle of hierarchical composition can be applied to arive a global priority for the elements in the lowest level with respect to the decision goal. Here is the adoption of the principle of hierarchical composition contributed by Thomas L. Saaty [1,pp. 76-78]

Let $H$ be a complete hierarchy with largest element $b$ and $h$ levels. Let $B_{k}$ be the priority matrix of the $k$ th level, $k=2, \ldots, h$. If $w^{\prime}$ is the priority vector of the pth level with respect to some element $z$ in the $(p-l)$ st level, then the priority vector $w$ of the qth level $(p<q)$ with respect to $z$ is given by

$$
w=B_{q} B_{q-1}, \ldots, B_{p+1} w^{\prime}
$$

and the priority vector of the lowest level with respect to the element $b$ is given by

$$
w=B_{h} B_{h-1}, \ldots, B_{2} w^{\prime}
$$

From the point view of system theory, a decision system which can be considered as a hierarchical structure and can be employ the principle of hierarchical composition to derive a global priority in the AHP, The following three condition for the system must be met: 
1. The elements in the system can be clustered as group or level. The elements in a same level have similar properties. The interdependence and mutual impact among the elements in a same level are too small to be considered.

2. There exists an element in the system which plays a core rule in the system. The element is called the goal of the system. The impact or function of the goal can be transformed into the elements in next lower level which transform the impact or function into the elements in another next lower level. The system function can be display in the entire hierarchical structure.

3. There are no feedback impact on any element came from other elements lower than it.

Otherwise, the principle must be adjusted according to the decision system. In fact, many decision problems have not pure hierarchical structure. There are interrelationships among elements within a group, i.e. inner dependence, or between groups, i.e. feedback relation. If both inner dependence and feedback relationship exist in a decision system, the system should considered as a general form rather than a hierarchical structure.

When a decision problem has been broke into basic decision elements, which can be clustered into groups, and local priorities of the elements in a group with respect to their related element have been derived, we can construct the supermatrix The supermatrix is an important tool in the priority setting of system with feedback. After the local priorities of the elements in level with respect to the $(j, l)$ th element level $j$ have been found as

$$
\left(w_{i, 1}^{(j, l)} \cdot w_{i, 2}^{(j, l)} \cdot \ldots \ldots w_{i, n_{i}}^{(j, l)}\right)^{T}
$$

we can construct the unweighted supermatrix W

$$
W=\left(\begin{array}{cccc}
W_{11} & W_{21} & \ldots & W_{1 N} \\
W_{21} & W_{22} & \ldots & W_{2 N} \\
\ldots & \ldots & \ldots . & \ldots \\
W_{N 1} & W_{N 2} & \ldots & W_{N N}
\end{array}\right)
$$

where 


$$
W_{i j}=\left(\begin{array}{cccc}
W_{i, 1}^{(j, 1)} & W_{i, 1}^{(j, 2)} & \ldots & W_{i, 1} \\
W_{i, 2}^{(j, 1)} & W_{i, 2}^{(j, 2)} & \ldots & W_{i, 2}^{\left(j, n_{j}\right)} \\
\ldots & \ldots . & \ldots & \ldots \ldots \\
W_{i, n_{i}}^{(j, 1)} & W_{i, n_{i}}^{(j, 2)} & \ldots & W_{i, n_{i}}^{\left(j, n_{j}\right)}
\end{array}\right)
$$

To get the weighted supermatrix, we should construct the weighting matrix

$$
A=\left(\begin{array}{cccc}
a_{11} & a_{12} & \ldots & a_{1 N} \\
a_{21} & a_{22} & \ldots & a_{2 N} \\
\ldots & \ldots & \ldots & \ldots \\
a_{N 1} & a_{N 2} & \ldots & a_{N N}
\end{array}\right)
$$

The weighted supermatrix can be calculated as

$$
\bar{W}_{i j}=\left\langle a_{i j} W_{i j}\right\rangle, \quad \bar{W}=\left\langle\bar{W}_{i j}\right\rangle
$$

To simplify the notion, we will use $W$ instead of $\bar{W}$ as the weighted supermatrix.

\section{The classification of systems with feedback}

According to the irreducible standard forms of supermatrix, the systems with feedback can be classified into the following four subclasses:

(1). Primitive system. The supernatrix of the primitive system is primitive. The system of Circular with Inner Dependence belongs to the primitive system. 
(2) Irreducible Circular System. The supermatrix of the irreducible circular system is not primitive but irreducible. The system of Circular with Inner Independence belongs to the irreducible circular system.

(3). Isolated-Block-Primitive System. The supermatrix of the isolated block primitive system is reducible but the isolated blocks in its reducible standard form are all primitive. The system with Hierarchical Structure without feedback among levels is the isolatedblock-primitive system.

(4). Isolated-Block-Imprimitive System. The supermatrix of the isolated-blockImprimitive system is reducible and one of isolated block is Imprimitive or circular. The system with Hierarchical Structure with feedback among levels is the isolated-blockImprimitive system.

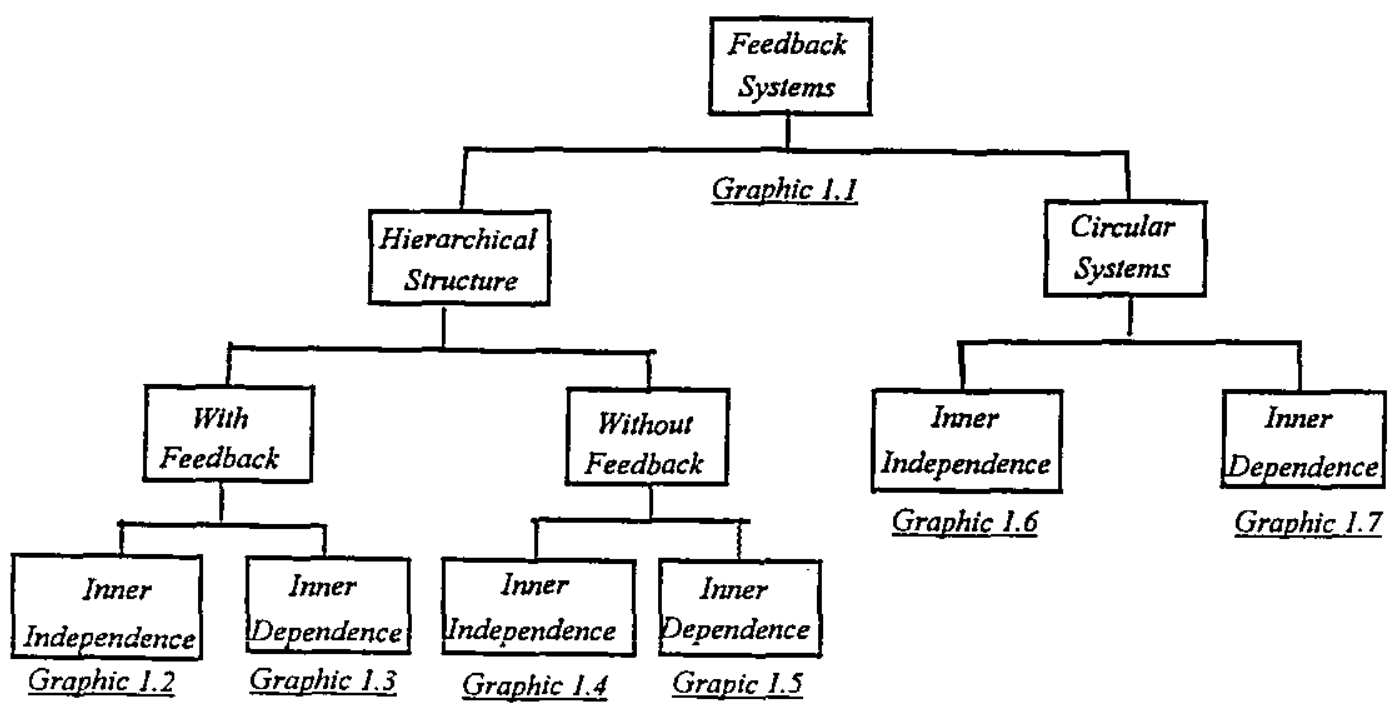



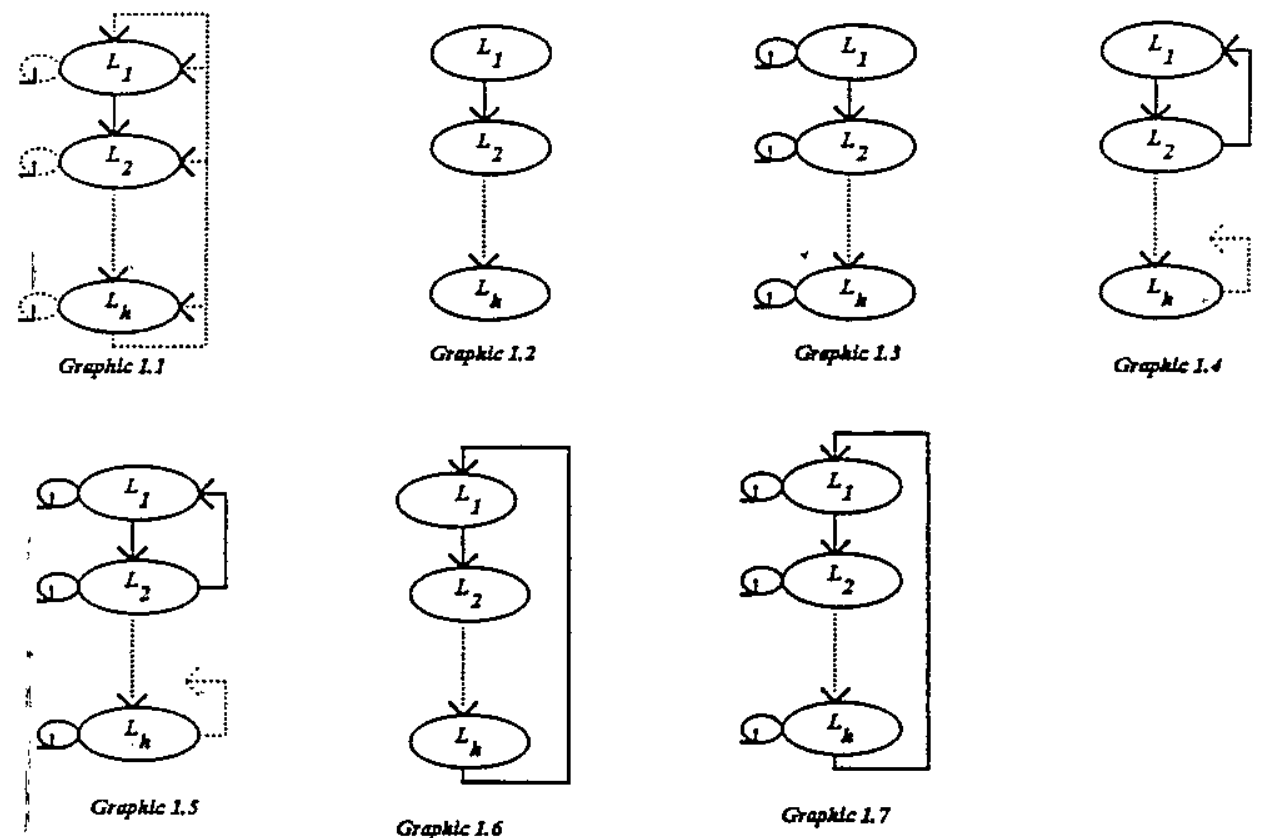

Grapile 1.7

Grople 1.6

Graphics 1.1-1.7: The classification of the feedback sustems

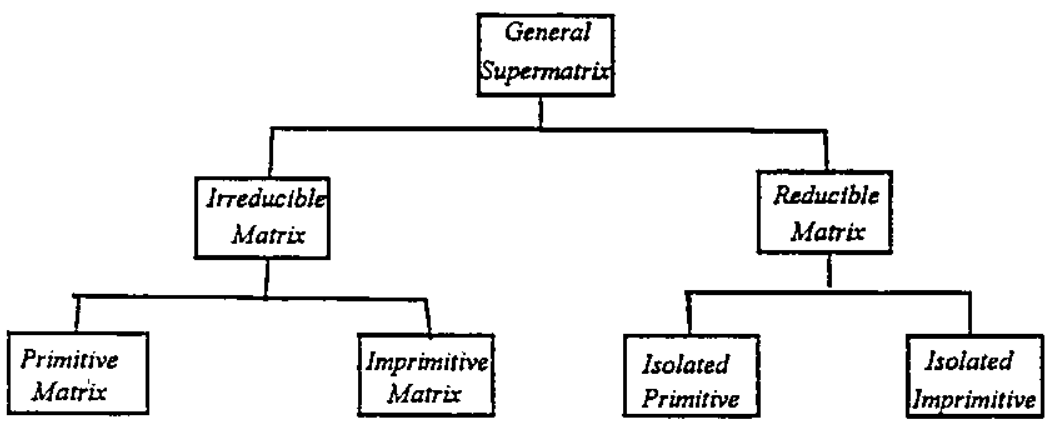

Graphic 2: The classification of the supermatrix

\section{The Limiting Impact Priority (LIP) and the Limiting Absolute Priority (LAP)}

Because of the existence of interdependence among elements and/or groups, we must consider the impact of the interdependence to priority setting. In this case, we do not usually have a top level as a frame of reference to carry out composition sequentially from level to level. The elements of the system can interact along more than a single path. In order for the measurement of priorities to be meaningful, there needs to be uniformity in how to consider all the paths. The priorities of any component of a system with respect to any other may be measured in a non-unique way along the paths and cycles which connect them. Instead of the hierarchical composition we mentioned early, the Limiting Impact 
Priority (LIP) and the Limiting Absolute Priority (LAP) should be considered and calculated in systems with feedback or interdependence.

The LIP is defined as $W^{\infty}$, if it exists. $W$ is the supermatrix above. The LIP gives a set of ultimate priorities of every element in the system with respect to each element measured by going around the cycle infinite times. It is the limiting state of the relative priority of every element in the system with respect to each element in that system. The LAP is defined as ${ }_{w}^{(\infty)}=w^{\infty}{ }^{(0)}$, where $w^{(0)}$ is the initial priority. If the LAP is independent of the initial priorities $w^{(0)}$, the independence is called the ergodicity of the system.

To understand the LIP and LAP better, we take the following feedback system as example.

Suppose A\$A College is going to hiring a new faculty. There are three candidates: $\mathbf{A}_{1}$, $A_{2}, A_{3}$. Criteria to evaluate the candidates are Teaching performance $\left(C_{1}\right)$, Research ability $\left(C_{2}\right)$, and Personal background $\left(C_{3}\right)$. After construct pairwise comparison, we calculate the local priority and supermatrix as follow:

$$
W=\left(\begin{array}{rrrrrr}
0.00 & 0.00 & 0.00 & 0.30 & 0.50 & 0.40 \\
0.00 & 0.00 & 0.00 & 0.40 & 0.10 & 0.20 \\
0.00 & 0.00 & 0.00 & 0.30 & 0.40 & 0.40 \\
0.25 & 0.10 & 0.40 & 0.00 & 0.00 & 0.00 \\
0.25 & 0.50 & 0.30 & 0.00 & 0.00 & 0.00 \\
0.50 & 0.40 & 0.30 & 0.00 & 0.00 & 0.00
\end{array}\right)
$$

which can be considered as the directed or one-step impact priorities of elements with respect to elements in the system. If we concern the impact transformation along two-steppaths, we need to calculate the priorities of elements with respect to elements along all possible two-step path in the system. It can be done by multiplication of $W$, i.e.

$$
W^{2}=\left(\begin{array}{cccccc}
0.40 & 0.44 & 0.39 & 0.00 & 0.00 & 0.00 \\
.225 & 0.17 & 0.25 & 0.00 & 0.00 & 0.00 \\
.375 & 0.39 & 0.36 & 0.00 & 0.00 & 0.00 \\
0.00 & 0.00 & 0.00 & .235 & .295 & .28 \\
0.00 & 0.00 & 0.00 & . .365 & .295 & .32 \\
0.00 & 0.00 & 0.00 & .400 & .410 & .40
\end{array}\right)
$$

Similarly, we can calculate the three-step path impact priorities as 


$$
W^{3}=\left(\begin{array}{llllll}
0.000 & 0.000 & 0.000 & .4130 & .4000 & .4140 \\
0.000 & 0.000 & 0.000 & .2105 & .2295 & .2140 \\
0.000 & 0.000 & 0.000 & .3765 & .3705 & .3720 \\
.2725 & .2830 & .2665 & 0.000 & 0.000 & 0.000 \\
.3250 & .3120 & .3305 & 0.000 & 0.000 & 0.000 \\
.4025 & .4050 & .4030 & 0.000 & 0.000 & 0.000
\end{array}\right)
$$

From the limiting supermatrices:

$$
\lim _{k \rightarrow \infty} W^{2 k+1}=\left(\begin{array}{cccccc}
0.00 & 0.00 & 0.00 & .405 & .405 & .405 \\
0.00 & 0.00 & 0.00 & .222 & .222 & .222 \\
0.00 & 0.00 & 0.00 & .373 & .373 & .373 \\
.273 & .273 & .273 & 0.00 & 0.00 & 0.00 \\
.324 & .324 & .324 & 0.00 & 0.00 & 0.00 \\
.403 & .403 & .403 & 0.00 & 0.00 & 0.00
\end{array}\right)
$$

and

$$
\lim _{k \rightarrow \infty} W^{2 k}=\left(\begin{array}{cccccc}
.405 & .405 & .405 & 0.00 & 0.00 & 0.00 \\
.222 & .222 & .222 & 0.00 & 0.00 & 0.00 \\
.373 & .373 & .373 & 0.00 & 0.00 & .0 .00 \\
0.00 & 0.00 & 0.00 & .273 & .273 & .273 \\
0.00 & 0.00 & 0.00 & .324 & .324 & .324 \\
0.00 & 0.00 & 0.00 & .403 & .403 & .403
\end{array}\right)
$$

the LAP $\stackrel{*}{P}$ and LIP of the three candidates are given by 


$$
W^{\infty}=\left[\lim _{k \rightarrow \infty} W^{2 k}+\lim _{k \rightarrow \infty} W^{2 k+1}\right] \div 2=\left(\begin{array}{llllll}
.405 & .405 & .405 & .405 & .405 & .405 \\
.222 & .222 & .222 & .222 & .222 & .222 \\
.373 & .373 & .373 & .373 & .373 & .373 \\
.273 & .273 & .273 & .273 & .273 & .273 \\
.324 & .324 & .324 & .324 & .324 & .324 \\
.403 & .403 & .403 & .403 & .403 & .403
\end{array}\right)(14)
$$

This means that the priorities of three candidates are $.273, .324, .403$. i.e. the third candidate has highest rank among the three candidates.

\section{Deriving the Limiting Impact Priority for the Hierarchical Systems with Inner Dependence}

The Hierarchical Systems with inner dependence can be characterized by the following properties:

1. The elements in the system can be clustered as several groups. The elements in each group have a similar attribute or characters as well as strong connection among them. The impact of the connection between the elements should not be ignored and must be counted when the global priorities are derived.

2. The system has a goal as the highest level which dominants the function of the system. The hierarchical dominance is only relationship among the level. Therefore the lowest level of alternative and the its elements do not dominant any other levels.

3. The system has no feedback dominant relationship among the levels.

The difference between the regular hierarchical structure (without inner dependence) and the inner dependent hierarchical structure is the interaction among the elements with a level. The principle of hierarchical composition is no longer applied for deriving the priority of the inner dependent hierarchical system. In this case, the procedure for deriving the LIP and LAP in a feedback system can be utilized. However, the inner dependent hierarchical system is a special kind of feedback system, the procedure for deriving should have some feather. In fact, there is a simplified procedure for the inner dependent hierarchical system to derive the LIP and LAP. We begin with the structure of the supermatrix of the inner dependent hierarchical system.

Suppose that the inner dependent hierarchical system has only one highest element, i.e. the goal. The supermatrix of the system is 


$$
W=\left(\begin{array}{ccllc}
W_{11} & 0 & \cdots & 0 & 0 \\
W_{12} & W_{22} & \cdots & 0 & 0 \\
\cdots & \cdots & \cdots & \cdots & \cdots \\
0 & 0 & \cdots & W_{N, N-1} & W_{N N}
\end{array}\right)
$$

Note that the priorities of elements in second level is a vector raher than a matrix, $W^{2}$ is

$$
\quad W^{2}=\left(\begin{array}{cllcc}
W_{11} & 0 & \cdots \cdots & 0 & 0 \\
W_{22} W_{21} & \left(W_{22}\right)^{2} & \ldots \ldots & 0 & 0 \\
\cdots & \cdots & \cdots \cdots & \cdots & \cdots \\
0 & 0 & \cdots \cdots & W_{N N} W_{N, N-1} & \left(W_{N N}\right)^{2}
\end{array}\right)
$$

Theorem

If the matrix $W_{N N}$ is normal, i.e. excepting 1 as his eigenvalue, there no exist any other eigenvalues the norm of which is equal to 1 , then $W_{N N}^{\infty}=\lim _{k \rightarrow \infty} W_{N N}^{k}$ exists and the LIP of the system is given by

$$
W^{\infty}=\left(\begin{array}{ccccc}
0 & 0 & \cdots \cdots & 0 & 0 \\
0 & 0 & \cdots \cdots & 0 & 0 \\
\cdots & \cdots & \cdots \cdots & \cdots & \cdots \\
D_{1} & D_{2} & \cdots \cdots & D_{N-1} & D_{N}
\end{array}\right)
$$

where

$$
\begin{gathered}
D_{N}=W_{N N}^{\infty} \\
D_{N-1}=D_{N} W_{N N-1}\left(I-W_{N-1 N-1}\right)^{-1}
\end{gathered}
$$




$$
\begin{gathered}
D_{i}=D_{i+1} W_{i+1 i}\left(I-W_{i i}\right)^{-1} \\
D_{2}=D_{3} W_{32}\left(I-W_{22}\right)^{-1} \\
D_{1}=D_{2} W_{21}\left(I-W_{11}\right)^{-1}
\end{gathered}
$$

Proof:

Since the sum of each column in $W_{N N}$ is $1, W_{N N}$ has 1 as the maximum eigenvalue. It is only eigenvalue there are no any other eigenvalue which has norm 1 as the norm for its eigenvalue. Therefore, the supermatrix of the system is the primitive or isolated-block primitive system. Hence $W_{N N}^{\infty}$ exists.

Note that for weighting matrix $A$, each of $a_{11}, a_{22}, \ldots, a_{N-1 N-1}$ is less than 1 . Therefore, the sum of each column of $W_{11}, W_{22}, \ldots, W_{N-1 N-1}$ is less than 1 and its spectrum radius is also less than 1 , i.e.

$$
\alpha\left(W_{i i}\right)<1 \quad i=1,2, \ldots, N-1
$$

So that,

$$
W_{i i}^{\infty}=\lim _{k \rightarrow \infty} W_{i i}^{k}=0 \quad i=1,2, \ldots, N-1
$$

We use mathematical induction to prove (17). When $\mathrm{i}=2$, the weighted supermatrix is given

$$
W=\left(\begin{array}{cc}
W_{11} & 0 \\
W_{21} & W_{22}
\end{array}\right)
$$

and

$$
W^{n}=\left(\begin{array}{cc}
W_{11}^{n} & 0 \\
Q_{21} & W_{22}^{n}
\end{array}\right)
$$


where

$$
Q_{21}=\sum_{p=0}^{n-1} W_{22}^{p} W_{21} W_{11}^{n-p-1}
$$

is a nonnegative matrix.

Since $\left\|W_{22}^{p}\right\|_{1}=1, W_{21}$ is constant matrix, for any given positive integer $\mathrm{p}$, there exists a positive matrix $M$ such that

$$
M>W_{22}^{p} W_{21}
$$

Therefore,

$$
\lim _{p \rightarrow \infty} W_{22}^{p} W_{21}=W_{22}^{\infty} W_{21}
$$

From

$\left(W_{11}^{n-1}+W_{11}^{n-2}+\ldots \ldots+W_{11}^{2}+W_{11}+I\right)\left(I-W_{11}\right)=1-W_{11}^{n}$
we have
$\lim _{n \rightarrow \infty}\left(W_{11}^{n-1}+W_{11}^{n-2}+\ldots . .+W_{11}^{2}+W_{11}+I\right)\left(I-W_{11}\right)=\lim _{n \rightarrow \infty}\left(I-W_{11}^{n}\right)\left(I-W_{11}\right)=\left(I-W_{11}\right)^{-1}$

Let $]=i n t[n / 2\}$, it implies

$$
\begin{aligned}
\lim _{n \rightarrow \infty} Q_{21} & =\lim _{n \rightarrow \infty} \sum_{p=0}^{n-1} W_{22}^{p} W_{21} W_{11}^{n-p-1}= \\
& =\lim _{n \rightarrow \infty}\left[\sum_{p=0}^{l} W_{22}^{p} W_{21} W_{11}^{n-p-1}+\lim _{n \rightarrow \infty} \sum_{p=l+1}^{n-1} W_{22}^{p} W_{21} W_{11}^{n-p-1}\right.
\end{aligned}
$$

From (22), it implies that 


$$
\begin{aligned}
& \sum_{p=0}^{l} W_{22}^{p} W_{21} W_{11}^{n-p-1}<M \sum_{p=0}^{l} W_{11}^{n-p-1}=M W_{11}^{n-l-1} \sum_{p=0}^{l} W_{11}^{l-p} \\
= & M W_{11}^{n-l-1}\left(I+W_{11}^{l+1}\right)\left(I-W_{11}\right)^{-1}
\end{aligned}
$$

It is clear that $l \rightarrow \infty,(n-l-1) \rightarrow \infty$ when $n \rightarrow \infty$. Since $\rho\left(W_{11}\right)<1$, it gets that

$$
\lim _{n \rightarrow \infty} W_{11}^{n-l-1}=\lim _{n \rightarrow \infty} W_{11}^{l+l}=0
$$

Hence the first part of (26) equals to 0, i.e.

$$
\lim _{n \rightarrow \infty} \sum_{p=0}^{l} W_{22}^{p} W_{21} W_{11}^{n-p-1}=0
$$

From (25), the second part of (26) is

$$
\begin{gathered}
\lim _{n \rightarrow \infty} \sum_{p=l+1}^{n-1} W_{22}^{p} W_{21} W_{11}^{n-p-1}=\lim _{n \rightarrow \infty} W_{22}^{\infty} W_{21} \sum_{p=l+1}^{n-1} W_{11}^{n-p-1} \\
W_{22}^{\infty} W_{21} \lim _{n \rightarrow \infty} \sum_{p=0}^{(n-l-1)-1} W_{11}^{(n-l-1)-p}=W_{22}^{\infty} W_{21}\left(I-W_{11}\right)^{-1} .
\end{gathered}
$$

i.e.

$$
\lim _{n \rightarrow \infty} Q_{21}=W_{22}^{\infty} W_{21}\left(I-W_{11}\right)^{-1}
$$

Therefore

$$
\bar{W}=\left[\begin{array}{cc}
0 & 0 \\
D_{1} & D_{2}
\end{array}\right], \quad D_{2}=W_{22}^{\infty}, \quad D_{1}=D_{2} W_{21}\left(I-W_{11}\right)^{-1}
$$

So that the statement is true for $n=2$. By inductive assumption, the statement is true when $\mathrm{k}=\mathrm{N}$. Let 


$$
\begin{aligned}
& W=\left(\begin{array}{cc}
Q_{11} & 0 \\
Q_{21} & Q_{22}
\end{array}\right), \quad Q_{11}=W_{11}, \quad Q_{21}=W_{21} \\
& Q=\left(\begin{array}{cccc}
W_{22} & 0 & \cdots & 0 \\
W_{32} & W_{33} & \cdots & 0 \\
\cdots & \cdots & \cdots & \cdots \\
0 & 0 & W_{N+1, N} & W_{N+1 N+1}
\end{array}\right)
\end{aligned}
$$

Note that $\mathrm{Q}_{22}$ is a NxN block supermatrix. By the inductive assumption, it is true for

$$
Q_{22}^{\infty}=\left(\begin{array}{ccccc}
0 & 0 & \cdots \cdots & 0 & 0 \\
0 & 0 & \cdots \cdots & 0 & 0 \\
\cdots & \cdots & \cdots \cdots & \cdots & \cdots \\
D_{1} & D_{2} & \cdots \cdots & D_{N-1} & D_{N}
\end{array}\right)
$$

Since $\rho\left(Q_{11}\right)=\alpha\left(W_{11}\right)<1<1$, using the procedure to prove the case for $\mathrm{k}=2$, we conclude that

$$
W^{\infty}=\left(\begin{array}{cc}
0 & 0 \\
Q_{22}^{\infty} Q_{21}\left(I-Q_{11}\right)^{-1} & Q_{22}^{\infty}
\end{array}\right)=\left(\begin{array}{cc}
0 & 0 \\
D_{22}^{\infty} W_{21}\left(I-W_{11}\right)^{-1} & D_{22}^{\infty}
\end{array}\right)
$$

$$
W^{\infty}=\left(\begin{array}{ccccc}
0 & 0 & \cdots \cdots & 0 & 0 \\
0 & 0 & \cdots \cdots & 0 & 0 \\
\cdots & \cdots & \cdots \cdots & \cdots & \cdots \\
D_{1} & D_{2} & \cdots \cdots & D_{N} & D_{N+1}
\end{array}\right)
$$

It means that the statement is true for $\mathrm{N}+1$. The proof of the theorem completes.

If there is only one element in the first level, i,e, there is only one goal for the hierarchical system the supermatrix for the system is little different from (15). It should be 


$$
W=\left(\begin{array}{ccclc}
0 & 0 & \cdots & 0 & 0 \\
b_{2} & W_{22} & \cdots & 0 & 0 \\
\cdots & \cdots & \cdots & \cdots & \cdots \\
0 & 0 & \cdots & W_{N, N-1} & W_{N N}
\end{array}\right)
$$

The LIP of the system is given by (17)-(21) and (45) as follows:

$$
D_{1}=D_{2} b_{2}
$$

If the ith level is independent, $W_{i i}$ should be substitute by 0 excepting $W_{n n}$ which should be $I$. For the regular hierarchical structure, we can obtain the same result with (1) and (2) by (17)-(21) and (45), i.e. the priorities of the alternatives with respect to the elements in the $i$ th level, $D_{i}$ is given by

$$
D_{i}=D_{i+1} W_{i+1, i}, \quad D_{N}=I, \quad i=1,2, \ldots, N-1
$$

\section{An numerical example of Inner Dependent System.}

Suppose that we are going to invest in one of three stocks: $A_{1}, A_{2}$ and $A_{3}$ according to three criteria which are $C_{1}$ : higher annual return, $C_{2}$ : lower risk and $\mathrm{C}_{3}$ : good reliability. We understand that the criteria and alternatives are inner dependent. A higher annual return of the stock may lead to a higher risk. A stock having a good reliability in present time will influence to reliability of other stock. We consider the decision problem as an inner dependent system. The structure of the problem can be shown as figure 6.1.

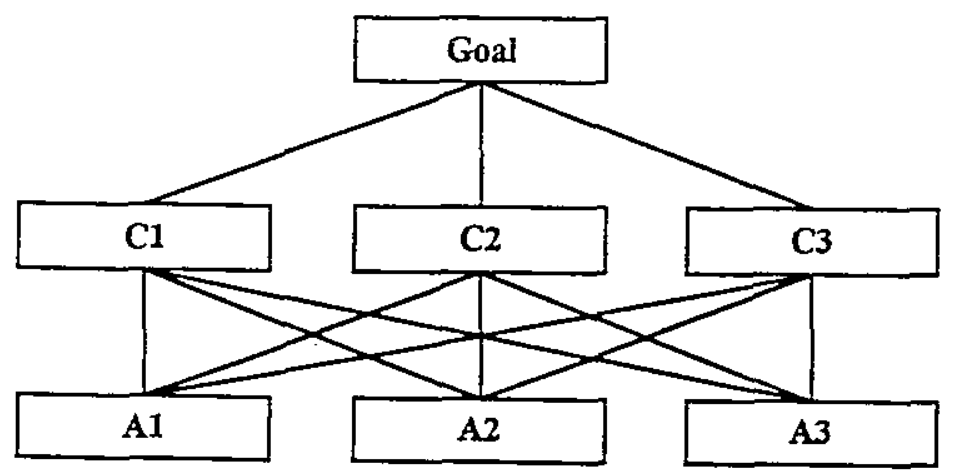

Figure 6.1 
The priorities of criteria with respect to the goal and alternatives with respect to different criteria are given as following matrices.

The priority vector of criteria with respect to the goal is

$$
\mathrm{W}_{12}=(0.6,0.3,0.1)^{\mathrm{t}}
$$

The priority matrix of alternatives with respect to criteria is

$$
W_{32}=\left[\begin{array}{lll}
.4 & 0.4 & 0.2 \\
.3 & 0.2 & 0.4 \\
.3 & 0.4 & 0.4
\end{array}\right]
$$

The inner dependent priority matrices are

$$
\begin{aligned}
& W_{22}=\left[\begin{array}{lll}
.7 & 0.1 & 0.15 \\
.2 & 0.7 & 0.15 \\
.1 & 0.2 & 0.7
\end{array}\right] \\
& W_{33}=\left[\begin{array}{lll}
.7 & 0.1 & 0.15 \\
.2 & 0.7 & 0.05 \\
.1 & 0.2 & 0.8
\end{array}\right]
\end{aligned}
$$

The weights for $W_{22}$ and $W_{32}$ are 0.6 and 0.4 , respectively.

The final LIP of alternative with respect to the goal are $0.31,0.19$ and 0.50 . According to the result, the best stock to invest is the first one. Comparing to the result of inner independent criteria and alternative, which is normal structure in the AHP: $0.38,0.28$ and 0.34 , and the result of inner dependent alternatives and inner independent criteria: 0.36 , 0.29 and 0.35 , we may see the influence of the inner dependence.

\section{REFERENCES}

[1] Thomas L. Saaty 1980 The Analytic Hierarchy Process, McGraw-Hill International Book Company 